\title{
La gestione dell'architettura civile e militare a Palermo tra XVI e XVII secolo: gli ingegneri del regno
}

\author{
M. Sofía Dı FEDE *
}

\begin{abstract}
SINTESI
ABSTRACT

La necessità di ammodernare e potenziare le cinture di fortificazioni dei centri più importanti della Sicilia costítuisce nel Cinquecento una delle principali preoccupazioni dei viceré; ne conseguirà un diretto coinvolgimento dell'autorità monarchica e dei suoi tecnici nell'attività costruttiva e nella riconfigurazione degli insediamenti urbani, in particolare della

capitale Palermo. Gli ingegneri regi coinvolti in tali vicende, talvolta, erano costretti a spostarsi frequentemente, per motivi professionali, allinterno dei dominii spagnoli, determinando spesso solo brevi

presenze in Sicilia; ma nel caso di permanenze più durature $i$ tecnici potevano accedere alla carica di "Ingegnere della Regia Corte", che obbligava però alla residenza nell'lsola. Le capacità richieste erano essenzialmente legate alla realizzazione e alla manutenzione delle strutture difensive, ma è ovvio che dovessero coprire l'intero ambito dell'attività architettonica vicereale; ció è evidente nel caso della capitale
\end{abstract}

* Dottore di Ricerca in Storia dell'Architettura e Conservazione dei Beni Architettonici Dipartimento di Storia e Progetto nell'Architettura, Università degli Studi di Palermo. 
Palermo, che imponeva l'esigenza di realizzare non solo indispensabili infrastrutture militari e civili, ma anche operazioni architettoniche e urbanistiche dalle valenze prettamente simboliche e monumentali. In tal senso il cantiere del

Palazzo Reale offre uno spaccato estremamente interessante sull'organizzazione dei ruoli tecnici e sulle diverse professionalità al servizio dell'autorità viceregia e, in particolare, sull'attività degli ingegneri regi a Palermo fra XVI e XVII secolo. erect basic civil and military infrastructure but also to pursue urban and architectural programs of a symbolic and monumental nature. In this way the works of the Royal Palace offer a view of extreme interest into the organization of the various professional and technical abilities of the engineers active in the viceregal service in Palermo in the $16^{\text {th }}$ and early $17^{\text {th }}$ centuries.

Agli inizi del XVI secolo il ruolo acquisito dalla Sicilia come avamposto dei dominii asburgici nel Mediterraneo rendeva indispensabile un totale ripensamento del sistema difensivo dell'isola, del tutto inadeguato a sostenere l'attività strategica della corona spagnola nei confronti dell'impero turco.

La conseguente necessità di ammodernare e potenziare le cinture di fortificazioni dei centri più importanti dell'isola costituirà, di fatto una delle principali preoccupazioni dei viceré che si risolverà, concretamente, in un direito coinvolgimento dell'autorità monarchica e dei suoi tecnici nell'attività costruttiva e nella riconfigurazione degli insediamenti urbani, soprattutto nel caso delle città maggiori, come Messina e Palermo ${ }^{1}$.

Riguardo quest'ultima, in particolare, a differenza del secolo precedente in cui erano state le iniziative private a segnare il passo del rinnovamento edilizio delle città, sarà soprattutto la committenza pubblica a gestire le trasformazioni urbane, all'interno della quale l'azione vicereale muterà gradualmente durante il Cinquecento le sue strategie d'intervento, partendo inizialmente da un interesse preponderante, peraltro obbligato dalla specifica situazione isolana, verso il settore delle fortificazioni

\footnotetext{
E' noto come l'opera di ammodernamento delle strutture difensive abbia preceduto e spesso contribuito a innescare processi di rinnovamento urbano talvolta sostanziali nelle città dell'lsola. Riguardo l'incidenza delle ragioni difensive sull'attività urbanistica siciliana del Cinquecento si veda in particolare A. CASAMENTO, II carattere militare dell'urbanistica del '500 in Sicilia, "Atlante di Storia Urbanistica Siciliana», n.5, 1982, págs. 7-16. Più in generale sul problema delle fortificazioni siciliane si vedano: R. SANTORO, Fortificazioni bastionate in Sicilia ( $X V$ e XVI sec.), in «Archivio Storico Siciliano", 1978, págs. 169-253; M. GIUFFRE, Castelli e luoghi forti di Sicilia, XII-XVII secolo, Palermo 1980; S. BoscaRinO, Architettura e urbanistica dal Cinquecento al Settecento, in Storia della Sicilia, vol. V, Napoli 1981, págs. 335-450; S. MAZZARELLA, R. ZaNCA, /l libro delle torri. Le torri costiere di Sicilia nei secoli XVI - XX, Palermo 1985; F. Russo, La difesa costiera del Regno di Sicilia, 2 voll., Roma 1992.
} 
per sviluppare gradualmente anche un'attenzione per la promozione artistica e architettonica che negli ultimi decenni del secolo diverrà, infine, prevalente.

Peraltro è innegabile che soprattutto nella prima metà del Cinquecento l'architettura militare sembri assorbire quasi totalmente le capacità propositive e le risorse economiche dell'amministrazione, ma è anche vero come tale attività prenda risalto in virtù dell'esistenza di una documentazione certamente più nutrita rispetto ad altri settori: gran parte della corrispondenza ufficiale tra i viceré e il sovrano, infatti, è dedicata ai problemi difensivi dell'Isola ${ }^{2}$, mentre non si fa menzione di tante altre iniziative intraprese dai viceré anche piuttosto impegnative, che però, probabilmente, alla corte di Madrid interessavano meno e, pertanto, potevano essere gestite autonomamente.

Questo può, forse, in parte spiegare il persistere di certe lacune storiografiche che continuano a pesare sull'architettura del Cinquecento in Sicilia e su alcuni dei suoi protagonisti. Rimane ancora tutta da indagare, per esempio, fatta eccezione per alcuni interventi nelle opere di fortificazione di Messina e Palermo, l'attività siciliana di Domenico Giunti ${ }^{3}$, chiamato nell'isola da Ferrante Gonzaga, viceré dal 1535 al 1546; lo stesso ingegnere toscano, nella sua biografia, afferma di avere realizzato a Palermo «...per il Suo Signore, et altri Principi, Palagi, Giardini Fontane, et altre opere mirabili... ${ }^{4}$, di cui non ci è pervenuta alcuna concreta notizia ${ }^{5}$. Di contro è ben nota l'opera di Antonio Ferramolino chiamato, fra

\footnotetext{
a Ci riferiamo, in modo particolare, alla parte dedicata al governo della Sicilia nel fondo "Estado», custodito presso l'Archivo General de Simancas (Valladolid), dove è contenuta gran parte di tale corrispondenza.

Sull'attività siciliana di Domenico Giunti (o Giuntalocchi) si vedano: G. Grosso CAcopARDI, Memorie storiche di Antonino Paolo Pllaja, Domenico Giuntalocchi e Giacomo del Duca, Messina 1842; A. Giuliana Alajmo, Architetti regi in Sicilia dal sec. XIII al sec. XIX, Palermo 1952, págs. 1213. L. SARullo, Dizionario degli artisti siciliani. Architettura, vol. I a cura di M.C. Ruggieri Tricoli, ad vocem, Palermo 1993, pág. 213. II Giunti, nel 1546, si trasferisce a Milano, al seguito di Ferrante Gonzaga, dove realizza, fra l'altro, la villa Simonetta e la chiesa di S. Angelo. Si vedano C. BARONI, Domenico Giunti architetto di don Ferrante Gonzaga e le sue opere in Milano, in "Archivio Storico Lombardo», n.s., III, 3-4, 1938, págs. 326-357; IDEM, Domenico Giunti architetto in Milano, in “Palladio», II, 1938, págs. 128-145.

4 La citazione è contenuta in M. GIUfFRÈ, Architettura e decorazione in Sicilia tra Rinascimento, Manierismo e Barocco, 1463-1650, in «Storia Architettura», IX, n. 1-2, 1986, pág. 20.

5 Secondo quanto indicato dalla documentazione, a Palermo il Giunti si sarebbe dedicato alla ristrutturazione del Castello a mare, in quegli anni residenza viceregia, e, probabilmente, alla realizzazione di un "sollazzo» per Ferrante Gonzaga, che recenti studi hanno permesso di individuare nella villa di Luca Cifuentes alle Croci. E. NeIL, La città verde: i giardini di Palermo, relazione tenuta durante i lavori del convegno di studi su L'urbanistica del Cinquecento in Sicilia, Roma 30-31 ottobre 1997, Facoltà di Architettura dell'Università di Roma «La Sapienza», i cui atti sono in corso di pubblicazione.
} 




Fig. 1. Palermo. Pianta della città dal Teatro geografico antiguo y moderno del Reyno de Sicilia (ms. del 1686, Biblioteca Nazionale di Madrid-Ministero degli Affari Esteri di Madrid), pubblicato in V. CONSOLO, C. DE SETA, Sicilia Teatro del Mondo, Torino 1990, pág. 249, tav. 37. Nell'immagine è ben visibile la cintura bastionata e la croce delle strade Toledo e Maqueda; in alto il complesso di Palazzo Reale, in basso l'antico porto della Cala e il Castello a mare. 
l'altro, a progettare un nuovo sistema di fortificazioni per la capitale dell'Isola ${ }^{6}$.

D'altronde è difficile immaginare che un personaggio come Ferrante Gonzaga, cresciuto tra i fasti della corte mantovana, 7 potesse limitarsi a svolgere il suo mandato da austero funzionario del re, senza intraprendere altre imprese architettoniche che non riguardassero il rinnovamento delle fortificazioni, nonostante egli stesso abbia voluto sottolineare tale aspetto, peraltro fondamentale nella sua attività di governo ${ }^{8}$.

E' chiaro come ai viceré interessasse evidenziare la propria capacità di assolvere ai compiti loro assegnati dalla corona, piuttosto che dare notizia di un mecenatismo artistico rivolto a soddisfare esigenze personali; quindi solo quando le iniziative architettoniche servivano a dare lustro all'autorità viceregia trovavano puntualmente un'immediata divulgazione.

La difesa del territorio costituiva, comunque, una preoccupazione costante per la monarchia spagnola non limitata specificatamente alla situazione siciliana ma estesa a tutti i suoi dominii, che da una parte provocava continui spostamenti da un paese all'altro dei tecnici della Corona, dall'altra rendeva necessario un continuo reclutamento di esperti nel settore delle fortificazioni provenienti dalle regioni più diverse, determinando un flusso costante tra la sede centrale e le periferie amministrative: è il caso, per esempio, di Tiburzio Spannocchi che nel 1580 viene inviato a Madrid dal

\footnotetext{
- Per l'occasione il Ferramolino stilò una relazione dettagliata in cui non solo venivano indicate le opere architettoniche da realizzare ma venivano fornite anche una serie di direttive sull'organizzazione della difesa, dalle artiglierie alle truppe e agli approwiggionamenti. A. Ferramolino, L'ordini di la fortificationi di quista felichi chita di Palermo..., in V. DI GIovanN, Le fortificazioni di Palermo nel secolo XVl giusta l'ordini dell'ing. Antonio Ferramolino ..., in «Documenti per servire alla Storia di Sicilia", s. IV, vol. IV, Palermo 1896. II Ferramolino è attivo al servizio del viceré Gonzaga anche in altri centri dell'isola, tra cui Messina dove si occupa dell'ammodernamento del sistema difensivo. Oltre agli studi sull'architettura militare siciliana già citati, si vedano: C. Proms, Biografie di ingegneri militari italiani dal secolo XIV alla metà del XVIII, «Miscellanea di Storia Italiana», 1874, págs. 369-373; A. GIULIANA ALAJMO, Architetti regi ..., cit., págs. 10-12; G. TADINI, Ferramolino da Bergamo. L'ingegnere militare che nel '500 fortificò la Sicilia, Bergamo 1977; M. FAGIOLO, M.L. MADONNA, II Teatro del Sole. La rifondazione di Palermo nel Cinquecento e l'idea della città barocca, Roma 1981, págs. 25-28.

G. GuerzonI, La corte gonzaghesca in età moderna. Struttura, ordini e funzioni, in S. BALBI DE CARo (a cura di), I Gonzaga. Moneta Arte Storia, catalogo della mostra - Mantova 1995, Milano 1995, págs. 90-96.

${ }^{8}$ Risulta emblematico, in tal senso, ciò che lo stesso viceré dice a proposito delle iniziative intraprese nella città di Palermo: «io l'ho circondata di bastioni che l'un vede l'altro, talmente che, accompagnata da un bellissimo sito piano, et per haver d'attorno assai buona muraglia, anchor che vecchia, io l'ho per inexpugnabile ...". F. GonzAGA, Relazione delle cose di Sicilia fatta... all'imperatore Carlo V, 1546, a cura di F. Carreri, in «Documenti per servire alla Storia di Sicilia», s. IV, vol. IV, 1896, pág. 7. Si veda anche G. CAPASso, /l Governo di Don Ferrante Gonzaga in Sicilia dal 1535 al 1543, in "Archivio Storico Siciliano», 1905, págs. 405-470; 1906, págs. 1-112 e 337-461.
} 
viceré Colonna dietro le pressanti sollecitazioni inviate dalla corona nel 1578 , per la preoccupante assenza nella penisola iberica di ingegneri competenti registrata già da alcuni anni ${ }^{9}$.

Accadeva più di frequente, però, che dalla Sicilia fosse richiesto l'intervento dei tecnici della Corona, talvolta anche sotto la forma di «pareri» inviati per dirimere le questioni più controverse, come nel caso, ad esempio, del tentativo di ampliare la città di Palermo in direzione nord ${ }^{10}$. In realtà molti ingegneri si spostavano all'interno dei dominii spagnoli al seguito delle armate sempre in continuo movimento tra un fronte di guerra e l'altro, determinando la temporaneità di alcune presenze nell'Isola, in particolar modo nel caso di militari di carriera, come Gabrio Serbelloni o Scipione Campi ${ }^{11}$.

9 A. CÁmARa Muñoz, Tiburzio Spannocchi, ingeniero mayor de los reinos de España, in “Espacio, Tiempo y Forma - Historia del Arte», s. VII, n. 2, 1988, págs. 77-90, in particolare págs. 77-78. L'ingegnere Spannocchi giunge in Sicilia alla fine degli anni settanta del Cinquecento; prima di trasferirsi in Spagna, dove condurrà una brillante carriera fino a ricevere la nomina di «ingegnere maggiore» della Corona nel 1601, realizza tra il 1577 e il 1578 una puntuale descrizione con relativa restituzione grafica delle coste isolane; it manoscritto, Descripción de las marinas de todo el Reino de Sicilia ..., ms. del 1596 conservato presso la Biblioteca Nacional de Madrid, n. 788, è stato di recente pubblicato in forma integrale: T. SpannoccHI, Marine del Regno di Sicilia, a cura di R. Trovato, Catania 1993. Oltre agli studi già citati si vedano: A. MAzzamuTo, Architettura e stato nella Sicilia del 500, "Atlante di Storia Urbanistica Siciliana", n. 8, 1986, págs. 12-65; L. DuFOuR, Atlante storico della Sicilia. Le città costiere nella cartografia manoscritta 1500-1823, Palermo 1992.

10 Tale ampliamento era stato deciso dal Consiglio civico nel marzo del 1568 con l'intento di accogliere dentro la cintura delle fortificazioni il nuovo porto, ma l'iniziativa, affidata operativamente al viceré d'Avalos, marchese di Pescara, accese un vivace dibattito che avrebbe percorso gli anni settanta del secolo tra posizioni pro e contro, da un iniziale consenso della corona spagnola esposto attraverso il «parere» favorevole, inviato da Madrid, dell'ingegnere Fratino (1571), attraverso i progetti espansionistici del Serbelloni (1572), il ridimensionamento proposto da Giulio Cesare Brancazio (1573), l'urgenza di completare le fortificazioni della città espressa dalla pianta di Alessandro Giorgi (1575), fino alla relazione di Scipione Campi (1576) che costituirà la premessa al definitivo abbandono del progetto. La vicenda è illustrata puntualmente in: M. GIUFFRE, Palermo "città murata" dal XVI al XIX secolo, in "Quaderno dell'I.D.A.U. Università di Catania", n. 8, 1976, págs. 41-68, in particolare págs. 49-55; IDEM, Castelli ..., cit., págs. 46-48; G. CARDAMONE, M. Giuffré, La città e il mare: il sistema portuale di Palermo, in G. Simoncini (a cura di), Sopra $i$ porti di mare, vol. III, Sicilia e Malta, Firenze 1997, págs. 159-192, in particolare págs. 170-175.

Il milanese Gabrio Serbelloni nel 1567 riceve l'incarico di ispezionare le fortificazioni di Napoli e Sicilia, dove in seguito viene nominato generale supremo del contingente militare stanziato nell'Isola. E' noto soprattutto per l'ambizioso progetto di ampliamento elaborato per la città di Palermo (vedi nota precedente) e l'analoga proposta formulata contemporaneamente per la città di Messina; inoltre si è occupato delle fortificazioni di Termini, Milazzo e Sciacca. L'ingegnere pesarese Scipione Campi giunge in Sicilia per redarre un resoconto sullo stato delle fortificazioni palermitane nel 1576 che indurrà ad abbandonare il progetto di ampliamento della città (vedi nota precedente); dopo avere prestato la sua opera a Trapani, Augusta, Siracusa e La Valletta, su richiesta di Gabrio Serbelloni verrà arruolato nell'esercito spagnolo ricevendo l'incarico di ingegnere maggiore del Regno di Spagna. Per i necessari riferimenti, oltre agli studi già citati sulle fortificazioni a Palermo $e$ in Sicilia, in particolar modo ai saggi di Maria Giuffrè, rimandiamo alle note biografiche e bibliografiche contenute in C. Promis, Biografie ..., cit., págs. 219-222 e 728-730, e in L. SARullo, Dizionario ..., vol. I, cit., págs. 397-398 e 81-82. 


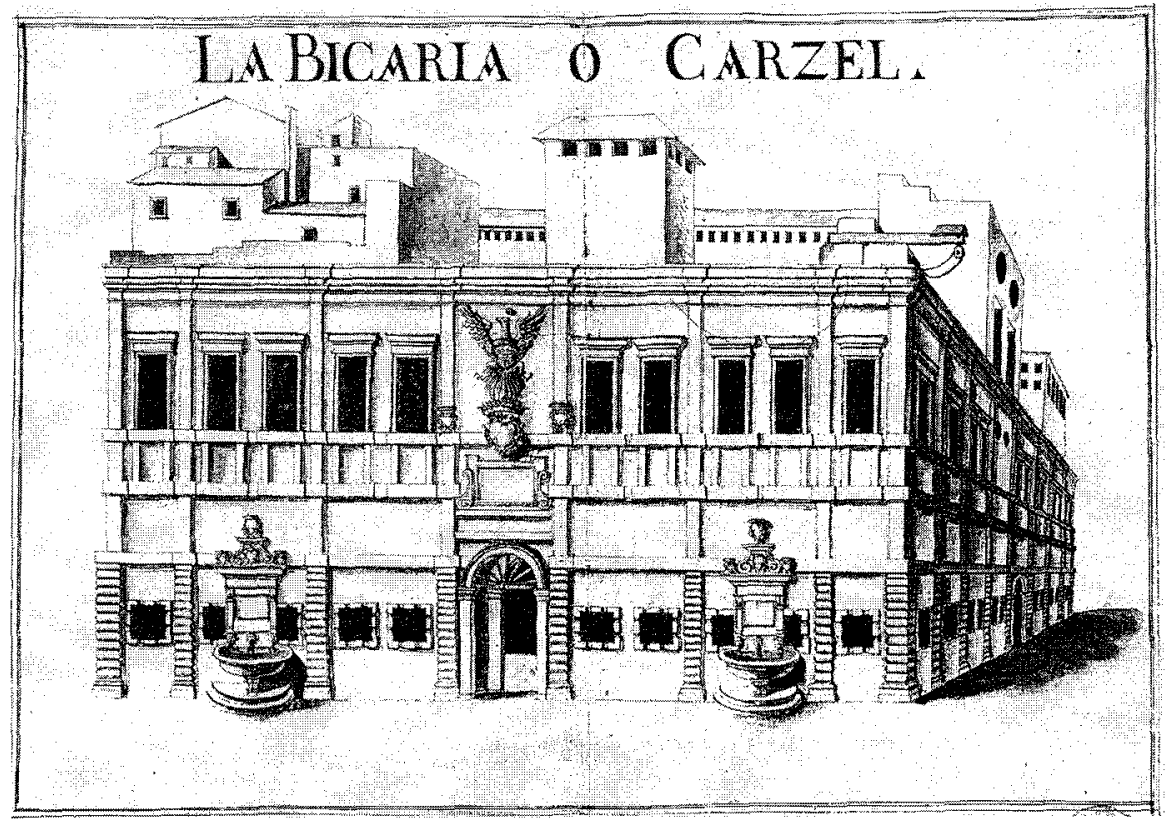

Fig. 2. Palermo. Palazzo della Dogana, poi della Vicaria dal Teatro geografico antiguo y moderno del Reyno de Sicilia (ms. del 1686, Biblioteca Nazionale di MadridMinistero degli Affari Esteri di Madrid), pubblicato in V. CONSOLO, C. DE SETA, Sicilia Teatro del Mondo, Torino 1990, pág. 255, tav. 42.

Verificandosi permanenze più durature, $i$ tecnici potevano accedere alla carica di «Ingegnere della Regia Corte» ${ }^{12}$ tramite il rilascio di una regolare patente che obbligava però alla residenza nel regno; le capacità richieste erano essenzialmente legate alla realizzazione e alla manutenzione delle strutture difensive, e tali competenze venivano ugualmente richieste ai tre titolari che detenevano la carica contemporaneamente, anche se la responsabilità ultima riguardo al sistema delle fortificazioni spettava all' «ingegnere maggiore ${ }^{13}$.

12 Non esistono studi specifici sulla figura dell'ingegnere regio in Sicilia, fatta eccezione per il saggio di A. GiulaAna ALAJMo, Architetti regi ..., cit., che, per quanto risulti ancora utile, andrebbe certamente aggiornato; rimandiamo, quindi, ai numerosi studi che abbiamo citato precedentemente.

13 Ciò si può dedurre chiaramente, ad esempio, da una «istruzione» inviata nel 1572 da don Carlo d'Aragona all' «ingegnere maggiore» del Regno Giovanni Antonio del Nobile: gli ordini sono tutti relativi ai probiemi della difesa dell'isola e spaziano dalla supervisione generale delle fortificazioni al controllo puntuale dell'attività dei cantieri aperti per verificare sia la qualità delle opere edificate sia la corretta gestione economica degli stessi, onde prevenire operazioni speculative, e, 
L'apparente uniformità di competenze richieste non è altrettanto riscontrabile nella formazione culturale dei personaggi che ebbero accesso a tale carica: a veri specialisti nel settore delle fortificazioni, come il Ferramolino, troviamo affiancati pittori-architetti, come il Giunti o successivamente lo Smiriglio ${ }^{14}$, e scultori-architetti come il Camiliani ${ }^{15}$, formatisi nelle botteghe e nei cantieri, a differenza di uno Spannocchi, architetto "colto" che accede alla pratica dell'architettura dallo studio della musica, della matematica, del disegno.

E' ovvio, peraltro, che gli incarichi assegnati agli ingegneri della Regia Corte non fossero limitati esclusivamente, per quanto prioritario, al settore delle fortificazioni, ma riguardavano l'attività architettonica di committenza vicereale nel suo complesso; la padronanza di un vasto raggio di competenze da parte dei tecnici della Corona risultava, del resto, indispensabile al momento in cui bisognava intervenire in una città come Palermo, il cui ruolo di capitale imponeva l'esigenza di realizzare non solo le infrastrutture necessarie alla gestione politico-economica del Regno e al funzionamento degli apparati governativi, ma anche operazioni, sia a carattere urbano sia a carattere strettamente architettonico, dalle forti valenze simboliche e monumentali.

infine, alla verifica dell'esatta corrispondenza tra quanto realizzato e le previsioni di progetto. La lettera di istruzioni è riportata in M. GIUFFRĖ, Palermo ..., cit., pág. 48; IDEM, Castelli ..., cit., pág. 43. Giovanni Antonio del Nobile, "todesco" di origine, è operativo in Sicilia come ingegnere maggiore dal 1564 al 1584 circa, come risulta dal regesto delle opere contenuto in C. FILANGERI, Aspetti di gestione ed aspetti tecnici nell'attuazione architettonica di Palermo durante il viceregno di Marcantonio Colonna (1577-1584), in R. CALANDRA (a cura di) Contributi al restauro architettonico e ambientale, Palermo 1978, págs. 48-49; per altre notizie, oltre ai saggi citati precedentemente, si veda la relativa scheda in L. SARULLO, Dizionario ..., vol. I, cit., pág. 137.

14 Sulla figura di Mariano Smiriglio rimandiamo alla nota 36.

16 Il toscano Camillo Camiliani giunge a Palermo nel 1574 per la collocazione e il completamento della fontana mamorea nel piano del Pretore, intraprendendo una carriera sempre più fortunata e diretta nei settori più diversi: dall'attività scultorea, che non abbandonerà mai, alla realizzazione di apparati effimeri, alt'architettura militare, quest'ultima in relazione alla nomina di architetto regio che riceverà ufficialmente nel 1586. G. SAMONÀ, L'opera dell'architetto fiorentino Camillo Camiliani in Sicilia alla fine del Cinquecento, in «Rivista del Regio Istituto di Archeologia e Storia dell'Arte», IV, 1932-33, págs. 227-278; A. Givliana Alajmo, Architetti ..., cit., págs. 16-17; A. CASAMENTO, I/ "Libro delle Torri maritime" di Camillo Camilliani (1584), in "Storia della Città», n.12-13, 1979, págs. 121-144; S. Mazzarella, R. Zanca, II libro ..., cit.; A. Mazzamuto, Architettura ...cit., págs. 66-80; S. Pedone, La fontana Pretoria, Palermo 1986; F. PAOLINo, Tre opere di Camillo Camiliani, in "Archivio Storico Messinese", n. 58, 1991, págs. 47-95; M. SCARLATA, L'opera di Camillo Camiliani, Roma 1993. Camillo Camiliani è attivo anche nel cantiere del Palazzo Reale di Palermo fin dai primi anni della sua permanenza nell'isola, sempre, però, in qualità di scultore; infatti, anche dopo aver ricevuto la patente di ingegnere regio, non ottiene incarichi direttivi all'interno del cantiere, ma gli viene commissionato il disegno dell'aquila marmorea da collocare nel portale centrale della nuova facciata del Palazzo nel 1600 e il disegno delle armi da collocare ai quattro angoli del cortile Grande nel 1602. Archivio di Stato di Palermo, Secrezia, vol. 1556, ff. 9091 ; vol. $1557,146$. 
In tal senso il trasferimento nel 1553 della sede vicerale nel Castello di S. Pietro, l'antica reggia normanna, e la conseguente opera di trasformazione della fabbrica nell'attuale Palazzo Reale, inaugura per la città di Palermo una stagione di febbrile attività architettonica e urbanistica che si protrarrà nei primi decenni del secolo successivo ${ }^{10}$, rispetto alla quale il cantiere del palazzo offre uno spaccato estremamente interessante sia sul circuito di artisti e maestranze legate alla Corte, che sull'organizzazione dei ruoli tecnici e sulle diverse professionalità al servizio dell'autorità viceregia.

In realtà sui primi interventi condotti nel complesso del Palazzo Reale, immediatamente dopo il trasferimento della sede vicereale, la documentazione è molto scarsa e fino a oggi non ha consentito di individuare l'autore del progetto di riconfigurazione dell'edificio; per tale ragione la notizia di una collaborazione tra Bustamante de Herrera e Juan de Vega, per risolvere il problema della fortificazione del Castello a mare, ${ }^{17}$ apre il campo a

16 II trasferimento della sede vicereale dal Castello a mare al Castello di S. Pietro fu determinato, concretamente, dagli acerbi conflitti sorti tra il viceré Juan de Vega e l'Inquisizione, che occupava dall'inizio del XVI secolo l'antica reggia normanna e che, di fatto fu "scacciata" d'autorità dalla prestigiosa residenza e trasferita forzatamente nel Castello a mare. Al di là delle ragioni contingenti, tuttavia, non può sfuggire il valore simbolico di una riappropriazione da parte del viceré spagnolo dei luoghi della tradizione monarchica siciliana, che, riproponendo l'antica dicotomia tra castello di «terra» e castello di «mare», renderà, in un certo senso, inevitabile la complessa operazione di rettificazione e prolungamento del Cassaro, avviata a partire dal 1567 durante il viceregno di Garçia di Toledo, da cui la nuova strada prenderà il nome. Intorno alla riconfigurazione del Palazzo Reale di Palermo nella seconda metà del Cinquecento e alle relazioni tra la vicenda del cantiere e il contemporaneo rinnovamento urbano della città abbiamo tenuto, di recente, una comunicazione dal titolo Architettura di stato a Palermo nella seconda metà del Cinquecento: le sedi del potere e la città, durante i lavori del convegno di studi su L'urbanistica del Cinquecento in Sicilia, cit. Più in generale, sulla vicenda urbana di Palermo tra Cinquecento e Seicento rimandiamo a: M. Giuffre', Palermo..., cit.; C. DE SETA, L. Di Mauro, Le città d'italia. Palermo, Bari 1980; M. Fagiolo, M.L. MadonnA, I/ Teatro ..., cit.; E. Guidoni, L'arte di costruire una capitale. Istituzioni e progetti a Palermo nel Cinquecento, in "Storia dell'Arte Italiana", Einaudi, Torino 1983, vol:XII, págs. 265-297; M.S. Di FEDE, Architettura e trasformazioni urbane a Palermo nel Cinquecento: la committenza viceregia, in «Espacio, Tiempo y Forma - Historia del Arte», s. VII, $n$. 8, 1995, págs. 103-117.

17 Da una lettera del 1547 di Juan de Vega a Carlo V: «Que en la fortificaçion de este Castillo de Palermo como su mag. ordena en su instruction he pensado por todas las vias algun remedio para labralle conforme al deseño q. su mag. ha embiado y en lo q. aca mas pareçiere conveniente y con el pareçer de ferramolino y del algunas otras personas de experiençia spero se hara tal obra y fabrica mediante la qual $q$. dara honestamente fortificado y podra servir en tiempo de necessidad porq. de la manera q. agora se halla serca para hazer poco serviçio y por no interesar el patrimonio real se haxan expender en la dicha fabrica algunos dineros extraordinarios de fiscalia $y$ se gastaran con todo buen recado y solamente en a quello $q$ sera mas util y necessario y tocara a la mera fortificaçion y no a otra comodita de estançias para habitaçion y del successo tendre confirmamente avisada a su mag: y q. bustamante de Herrera lleva al verdadero desegño de como agora esta el castillo y tiene mucha notiçia y practica de cosas de architectura y fortificaçion con el qual si su mag. fuere servido podra veer el desegno de el castillo q de aca lleva y mandar lo q. en el se oviere de hacer...». Archivo General de Simancas, Estado, legajo 1118, doc. 53, f. 1. 
nuove e suggestive ipotesi su una possibile paternità «spagnola» del programma di interventi previsti per il Palazzo Reale.

Anche la documentazione degli anni sessanta e settanta è molto oscura per quello che riguarda l'organizzazione e le gerarchie all'interno del cantiere, affidato, pare, a un «capomastro della regia curti», al quale era chiaramente demandato il compito di controllare la corretta esecuzione dei lavori ${ }^{18}$. Né, allo stato attuale degli studi, è chiaro se l'ingegnere Alessandro Giorgi, che interviene nella fabbrica nel 1577, ${ }^{19}$ costituisca una presenza transitoria nel cantiere o presti la sua opera in qualità di «capomastro del sacro regio palatio e dell'acque della regia corte», ${ }^{20}$ ruolo che avrebbe ricoperto successivamente Giovanni Antonio Salamone ${ }^{21}$.

Si deve a Marcantonio Colonna, viceré dal 1577 al 1583, la riorganizzazione amministrativa del cantiere, attraverso cui il ruolo degli ingegneri regi viene definitivamente chiarito. Nell'ordine rilasciato l't settembre 1579 il viceré stilava una normativa, rimandando al suo diretto controllo ogni iniziativa intrapresa nel cantiere ${ }^{22}$ e concentrando

Potrebbe trattarsi di Bartolomeo Bustamante, l'architetto gesuita che opera prevalentemente in Spagna tra gli anni quaranta e gli anni sessanta del Cinquecento, considerato dalla critica fra $i$ primi ad avere introdotto in Spagna il linguaggio classico ortodosso, derivato direttamente dai modelli italiani, che ebbe modo di conoscere direttamente grazie ai suoi numerosi viaggi nella Penisola; si veda in proposito: A. Rodríguez Gutieraez de Ceballos,. S.I., Bartolomé de Bustamante y los origenes de la architectura jesuítica en España, Roma 1967. É possibile peró che l'interlocutore di Juan de Vega sia il fratello di Bartolomeo, Jeronimo Bustamante de Herrera, conosciuto come l'autore dell'impianto urbano di Valdepeñas de Jaén: F. Marías, El largo siglo XVI, Madrid 1989, pág. 77. Jeronimo soggiorna lungamente a Roma, con qualche breve interruzione, dal 1543 al 1557, cioè nel periodo in cui Juan de Vega prima è ambasciatore di Carlo $V$ presso la Santa Sede (1543-1547), poi diventa viceré di Sicilia (1547-1557): IDEM, L'arquitectura del Renacimiento en Toledo (1541-1631), vol. 1, Toledo 1983, pág. 287; su Juan de Vega: Marqués del SaLtillo, Juan de Vega, embajador de Carlos V en Roma (1543-47), Madrid 1946.

${ }_{18}$ Nel contratto stipulato nel 1567 per la fabbrica della facciata a logge, poi non realizzata, si fa riferimento a un "capomastro della regia curti", il non meglio identificato «mastro ambroxio". il contratto è citato in A. GIuFfridA, La storia del Palazzo reale emerge dalle ricerche archivistiche, in "Cronache Parlamentari Siciliane», IV, n. 4-5, 1980, pág. 9.

${ }_{19}$ L'ingegnere interviene nel cantiere in merito a dissesti verificatisi nella loggia prospiciente il cortile della Fontana, ordinandone il puntellamento. Archivio di Stato di Palermo, Secrezia, vol. 1536, ff. 33-42.

${ }_{20}$ Il Filangeri cita la notizia a proposito della successione nell'incarico di Giovanni Antonio Salamone. C. FilangeRi, Aspetti di gestione ..., cit., pág. 49. Alessandro Giorgi, ingegnere della città di Palermo, è noto soprattutto per aver realizzato nel 1575 un progetto di revisione della cintura bastionata di Palermo, il cui grafico è custodito presso l'Archivo General de Simancas. M. GiufFrè, Palermo ..., cit., págs. 45-46.

${ }_{21}$ In un pagamento del 16 febbraio 1579 (Archivio di Stato di Palermo, Secrezia, vol. 1536, ff. 52-53) Giovanni Antonio Salamone viene definito con questo titolo. Il Filangeri ne ha ricostruito l'attività come ingegnere regio dal 1574 al 1583 in Aspetti di gestione ..., cit., pág. 49.

22 Dal documento traspare in maniera evidente l'insoddisfazione del viceré nei riguardi della gestione amministrativa del cantiere: le direttive che egli fornisce, infatti, sono tutte indirizzate a 




Fig. 3. Palermo. Porta Nuova, fronte verso Monreale, dal Teatro geografico antiguo y moderno del Reyno de Sicilia (ms. del 1686, Biblioteca Nazionale di Madrid-Ministero degli Affari Esteri di Madrid), pubblicato in V. CONSOLO, C. DE SETA, Sicilia Teatro del Mondo, Torino 1990, pág. 263, tav. 50.

nella figura dell'ingegnere della fabbrica ogni responsabilità direttiva ${ }^{23}$.

Istituita, quindi, ufficialmente la carica di ingegnere della fabbrica, in sostituzione del "capomastro del sacro regio palatio e dell'acque della regia corte», probabilmente senza grosse modifiche delle competenze

fornire un quadro preciso delle spese effettuate, anche quelle minime, per evitare abusi, alterazioni contabili e la sparizione di materiali acquistati per la fabbrica. Archivio di Stato di Palermo, Secrezia, vol. 1537, f. 24. II documento è citato anche in C. GuASTELLA, Ricerche su Giuseppe Alvino detto il Sozzo e la pittura a Palermo alla fine del Cinquecento, in Contributi alla storia della cultura figurativa nella Sicilia occidentale tra la fine del XVI e gli inizi del XVII secolo, Atti della giornata di studio su Pietro d'Asaro, Racalmuto - 15 febbraio 1985, Palermo 1985, pág. 77, nota 57.

23 All'ingegnere venivano così affiancati cinque «officiali»: il soprastante, con il compito di effettuare i pagamenti delle maestranze, staglianti e lavoratori a giornata; il provveditore, che doveva occuparsi dell'acquisto dei materiali e delle attrezzature per il cantiere; il «munizioniere», incaricato di custodire e immagazzinare tali materiali e attrezzature; il notaio della Secrezia, deputato alla cura de i libri contabili; infine riceveva un regolare stipendio anche un mastro d'ascia. Tutte le operazioni degli officiali dovevano essere compiute secondo le direttive dell'ingegnere, registrate 
tecniche richieste, questa veniva affidata ugualmente a Giovanni Antonio Salamone e anche in seguito sarebbe stato sempre un ingegnere della Regia Corte a dirigere il cantiere e a effettuare le stime dei lavori, con l'aiuto di un capomastro regolarmente stipendiato a partire dal $1584{ }^{24}$.

Rimane sempre molto complesso riuscire a stabilire, invece, la paternità dei progetti, poiché raramente la documentazione di cantiere o i contratti forniscono notizie in merito, né può essere sempre rimandata all'ingegnere della fabbrica; anzi accade più frequentemente il contrario, perché da una parte i responsabili del cantiere si trovavano spesso nelle condizioni di dovere condurre in porto interventi programmati precedentemente, dall'altra bisogna tenere conto dei possibili interventi da parte di altri tecnici al servizio della Corona.

Sebbene la direzione del cantiere sia rimasta affidata al Salamone fino al 1583, anno della sua morte, la presenza in Sicilia di Tiburzio Spannocchi ${ }^{25}$ nei primi anni del viceregno del Colonna farebbe ipotizzare un suo diretto coivolgimento nella cospicua attività architettonica e urbanistica promossa a Palermo dal principe romano, incluso il complesso del Palazzo Reale, anche se, in particolare, la sopraelevazione di Porta Nuova sembra riferibile con una certa sicurezza al Salamone ${ }^{26}$. Tuttavia l'unica opera che è possibile attribuire allo Spannocchi con qualche certezza è il palazzo della Dogana, poi della Vicaria, realizzato tra la via Toledo e l'antico porto della Cala a partire dal $1578^{27}$.

debitamente nei libri contabili e comunque essere controfirmate dal viceré per potere diventare esecutive, motivo per cui da quel momento in poi la documentazione riguardante la fabbrica diviene molto minuziosa e consente di seguire l'iter costruttivo della fabbrica con molta precisione.

${ }_{24}$ Dal mese di aprile del 1584 riceve un regolare stipendio di 5 onze al mese Baldassare Natale. Archivio di Stato di Palermo, Secrezia, vol. 1541, ff. 176-177.

25 Si veda la nota $n .9$.

${ }^{26}$ In un pagamento dell' 1 maggio 1584, infatti, si fa riferimento alla "...logetta novamente fatta sopra la porta aurea olim detta. porta nova de questa città [...] conforme il desegno fatto per il quondam mag.o Jo. Antonio Salamone olim ingignero della frabica de questo sacro regio palatio...". In S. Di Matteo, La Porta Nuova a Palermo, Palermo 1990, Documenti, págs. s.n..

${ }_{27}$ Nell'introduzione del suo manoscritto dedicato alla descrizione delle coste isolane l'ingegnere toscano scrive di avere fornito i disegni e aver seguito l'avvio dei lavori di una «casa Real" nella città di Palermo per dare una sede definitiva ai tribunali regi dove le magistrature civili, penali e del real patrimonio potessero custodire i propri archivi (T. SPANNOCCHI, Marine ..., cit., f. 5; anche in A. CÁmara Muñoz, Tiburzio Spannocchi ..., cit., pág. 78). Sono le testimonianze di Francesco Paruta e Nicolò Palmerino (Diario della Città di Palermo ... (1500-1613), "Biblioteca Storica e Letteraria di Sicilia», a cura di G. Di Marzo, serie I, vol. I, Palermo 1869, pág. 85) e di Vincenzo Di Giovanni (Palermo Restaurato, XVII sec., ed. a cura di M. Giorgianni e A. Santamaura, Palermo 1989, pág. 146) a consentire di identificare la fabbrica dello Spannocchi nel palazzo della Vicaria, portato successivamente a compimento sotto la direzione di Giovan Battista Collepietra. Ricordiamo che un'immagine dell'edificio è contenuta nel Teatro geografico antiguo y moderno del Reyno de Sicilia, in V. Consolo, C. De SeTA, Sicilia Teatro del Mondo, Torino 1990, pág. 255, tav. 42. 
Alla morte di Giovanni Antonio Salamone, nel ruolo di ingegnere della fabbrica di Palazzo Reale gli succede il toscano Giovan Battista Collepietra, il quale manterrà l'incarico ininterrottamnte fino al $1602^{28}$.

II Collepietra è il personaggio che, in un certo senso, monopolizza l'attività architettonica palermitana nell'ultimo ventennio del Cinquecento, anche se come ingegnere regio non manca di intervenire in altre città per problemi legati alle fortificazioni. A Palermo, come ingegnere della città e del Nuovo Molo interviene in molti cantieri avviati dalla municipalità, fra cui lo stesso palazzo del Senato, dove dirige i lavori di decorazione della Sala Grande; gli si deve inoltre la regìa di complessi apparati effimeri, cicli pittorici, opere scultoree, a dimostrazione di una competenza che va oltre la specificità architettonica e costruttiva ma è indirizzata verso ogni espressione artistica legata all'arte del disegno: è insomma l'interprete più efficace di una concezione del cantiere come reale sintesi delle arti ${ }^{29}$.

Tuttavia, nonostante le riconosciute capacità anche da parte dei suoi contemporanei e la ventennale carriera al servizio della Corona, al momento di realizzare il cortile Grande e lo scalone d'onore nel Palazzo Reale ${ }^{30}$, il viceré Maqueda ne affiderà la progettazione al suo connazionale Diego Sanchez ${ }^{31}$,

28 La presenza dell'ingegnere è registrata sistematicamente nei libri contabili del cantiere. Archivio di Stato di Palermo, Secrezia, voll. 1541-1557.

${ }^{29}$ II D'Ariano lo definisce «...huomo che con lungo e continuato studio, ha fatto acquisto di tutte quelle cognizioni, che a' somma perfezione dell'arte sua, Vitruvio desiderava; e con molto andar attorno, e vedere una gran parte non pur dell'Europa, ma dell'Asia e dell'Africa, ha raccolte insieme nella memoria, quasi tutte le bellezze e le meraviglie che sparse qua, e là rendono molti luoghi del mondo riguardevoli e famosi...». G. D'ARiAno, Arco trionfale fatto in Palermo nell'anno MDXCl/ per la venuta dell'llustrissimo ed Eccellen. Signor Don Henrico Guzman Conte d'Olivares viceré di Sicilia, Palermo 1592, pág. 7. Sul Collepietra non esistono studi specifici, quindi rimandiamo alle schede contenute in: A. GivlianA AlAJMo, Architetti regi ..., cit., págs. 18-23; C. FILANGERI, Aspetti di gestione ..., cit., págs. 9-12, nota 36; M. FAGIOLO, M.L. MAdonnA, // Teatro ..., cit., págs. 221-222, appendice VI; L. SARULLO, Dizionario ..., vol. !, cit., págs. 112-114.

30 La scelia di aggiungere questi nuovi corpi al complesso sembra sia strettamente legata alla decisione presa dal Maqueda di concentrare nel palazzo tutte le magistrature del Regno, che troveranno, appunto, alloggiamento negli ambienti realizzati intorno al grande cortile. La costruzione dei nuovi corpi di fabbrica fu intrapresa immediatamente dopo l'arrivo del viceré Maqueda a Palermo, nell'autunno del 1598, quando vennero commissionate le prime ventiquattro colonne da collocare nel «cortile grande", ma tra il 1599 e il 1600 furono stipulati gran parte dei contratti per l'avvio delle opere: si trattava di realizzare una corte quadrata, ampliando l'antico «baglio di S. Pietro» dal perimetro irregolare, demolendone i corpi verso S. Maria dell'tria, cioè verso est, in modo da potere realizzare intorno i nuovi uffici. A partire dl 1601, venne invece avviata la costruzione dello scalone d'onore, realizzato sia per rendere possibile un agevole accesso ai tre livelli del cortile, sia per consentire, con vantaggio non indifferente, l'ingresso diretto alla grande sala dei Parlamenti Generali del Regno. Archivio di Stato di Palermo, Secrezia, vol. 1554, f. 143 e ss.; vol. 1555, f. 151 e ss.; vol. 1557, f. 115 e ss.

31 In un pagamento rilasciato al marmoraro comasco Massimiano del Fossato it 21 luglio 1599 per la realizzazione delle colonne del secondo ordine del cortile si fa riferimento al «...disegno fatto p. l'ingignero Diego sanches...». Archivio di Stato di Palermo, Secrezia, vol. 1554, f. 93. 


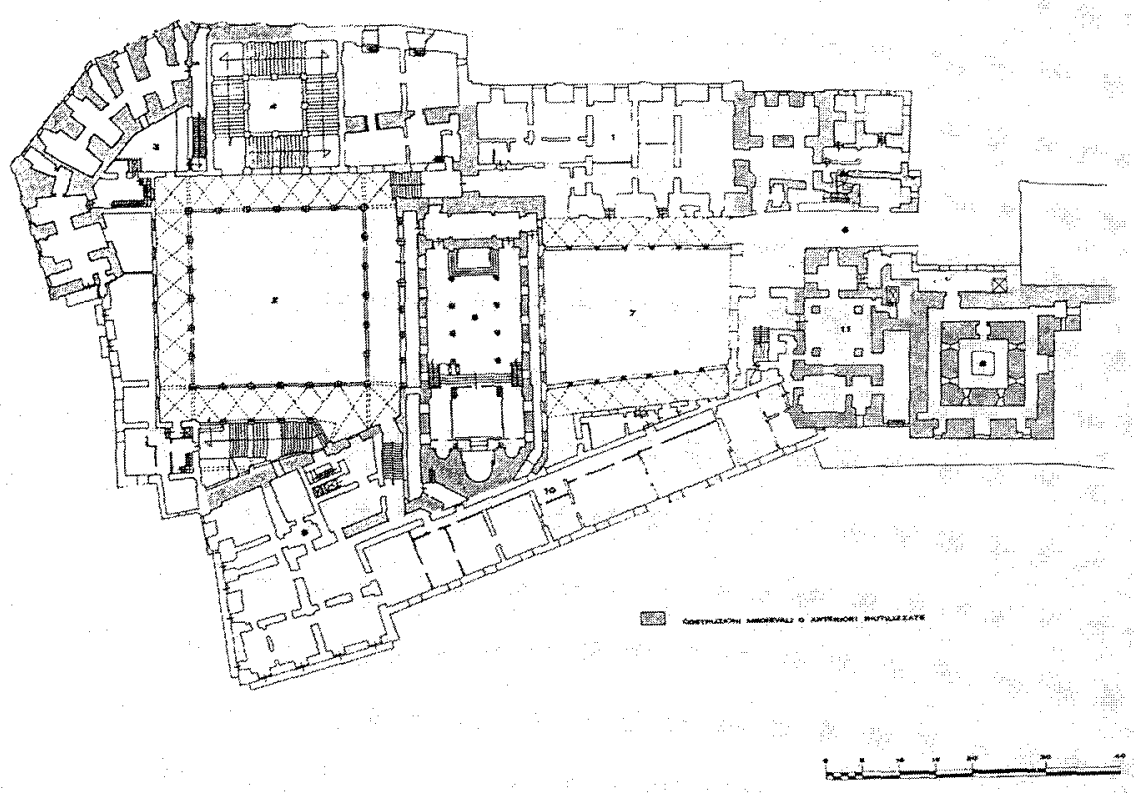

Fig. 4. Palermo. Palazzo Reale. Pianta al livello della Cappella Palatina. Sulla sinistra dell'immagine è visibile il cortile Maqueda con lo scalone d'onore in alto (Elaborazione grafica di R. Calandra e D. Ciriminna. Sta in Palazzo dei Normanni, Palermo 1991).

probabilmente giunto nell'isola al seguito del viceré castigliano, visto che non abbiamo notizie di una sua presenza nell'Isola nel periodo precedente ${ }^{32}$ : al di là dei ruoli ufficiali ricoperti nell'organizzazione tecnica e amministrativa dei lavori lo sviluppo della fabbrica veniva, di fatto, determinata, dalle personali scelte del viceré e dall'intervento di un tecnico di sua fiducia.

La possibilità di attribuire il progetto del cortile Grande (oggi cortile Maqueda) al Sanchez chiarisce, finalmente, le ragioni dell'evidente affinità esistente tra questo intervento nel Palazzo Reale di Palermo e talune so-

32 Fino a poco tempo fa era noto solamente che it Sanchez aveva operato come ingegnere regio fino al 1639 quando, per l'avanzata età, era stato sostituito nella carica da Gaspare Guercio; vedi A. Giuliana Alaumo, Architetti regi ..., cit., pág. 17. Piủ recentemente è stato documentato un suo intervento nel 1607 nel cantiere della Chiesa Madre di Milazzo; vedi E. D'AMico, Contributo allo studio dell'architettura dei secoli XVII e XVIIII a Milazzo sulla base di documenti inediti, in "Archivio Storico Messinese», 1978, págs. 153-189, in particolare pág. 160. 
luzioni adottate precedentemente in Spagna in occasione dei lavori di ammodernamento delle residenze reali, in particolar modo nell'Alcázar di Toledo, dove lo scalone d'onore è direttamente collegato al grande cortile colonnato, occupandone l'intero fronte opposto all'ingresso. Ovviamente la realizzazione palermitana mostra, comunque, alcune differenze sia nell'impianto dello scalone, che presenta uno sviluppo delle rampe più tradizionale, sia nella sovrapposizione dei tre ordini di archeggiature del cortile, configurati e dimensionati in maniera difforme l'uno dall'altro, per l'evidente necessità di doversi adattare ai vari livelli dei corpi di fabbrica preesistenti.

La documentazione rinvenuta sulla costruzione del cortile Maqueda, infine, getta nuova luce sopra un personaggio quasi del tutto sconosciuto come Diego Sanchez, dai connotati, tuttavia, ancora non facilmente valutabili; ciò in ragione anche del ruolo ambiguo da questi ricoperto nel cantiere di Palazzo Reale, di cui, pur contribuendo al prosieguo dei lavori almeno fino al $1609,{ }^{33}$ non assumerà mai la direzione, che invece, dopo il 1602 , sarà affidata a personaggi certamente non di secondaria importanza come gli ingegneri Orazio del Nobile, ${ }^{34}$ Giulio Lasso ${ }^{35} \mathrm{e}$, nel secondo decennio del Seicento, Mariano Smiriglio.

Quest'ultimo, infatti, nominato ingegnere del Senato palermitano nel 1602, ricoprirà anche la carica di ingegnere regio a partire dal $1610,{ }^{36}$ secondo una prassi già collaudata nel secolo precedente con Giovan Battista Collepietra e, probabilmente, ancora prima con Giovanni Antonio Salamone e Alessandro

33 Risalgono al 25 gennaio 1609 i capitoli per la realizzazione delle volte nel secondo ordine del loggiato del cortile Maqueda firmati da Diego Sanchez. Archivio di Stato di Palermo, Secrezia, vol. 1563 , ff. 6-7.

${ }_{34}$ Archivio di Stato di Palermo, Secrezia, voll. 1557-1559. Orazio del Nobile è attivo nell'Isola come ingegnere regio dal 1580 al 1610 , collaborando all'inizio della sua carriera con Giovanni Antonio del Nobile e Tiburzio Spannocchi; è noto per aver progettato la chiesa della Concezione di Palermo e avere condotto i primi lavori per il taglio della via Maqueda nella stessa città. Archivio Storico del Comune di Palermo, Raziocini, vol. XXXII, ff. 20 e ss.. Si veda anche A. GIULIANA Alajmo, Architetti regi ..., cit., págs. 14-16; C. FILANGERI, Aspetti di gestione ..., cit., págs. 50-51; V. SCUDERI, La chiesa dell'Immacolata Concezione a Palermo, Palermo 1994.

35 Su Giulio Lasso rimandiamo alla nota 38.

${ }_{36}$ In virtù di tali cariche lo Smiriglio si rivelerà come uno dei principali protagonisti della scena architettonica palermitana durante i primi decenni del XVII secolo. F. MELI, Degli architetti del Senato di Palermo nei sec. XVII e XVIII, in «Archivio Storico per la Sicilia», 1938-39, págs. 313322; A. Giuliana Alajmo, Architetti regi .... cit., págs. 15-16. Per un aggiornamento bibliografico sull'architetto si veda: G. CIOTTA, Mariano Smiriglio, architetto del Senato palermitano (1602-1636), in G. SPAgnesI (a cura di), L'architettura a Roma e in Italia (1580-1621), Atti del XXIII Congresso di Storia dell'Architettura - Roma 1988, vol. II, Roma 1989, págs. 387-393; M.S. DI FEdE, Mariano Smiriglio, architetto, in «Bollettino della Biblioteca», Dipartimento di Storia e Progetto nell'Architettura - Facoltà di Architettura di Palermo, n.2, 1993, págs. 75-80.

${ }_{37}$ C. FILANGERI, Aspetti di gestione ..., cit., pág. 30, nota 78. 


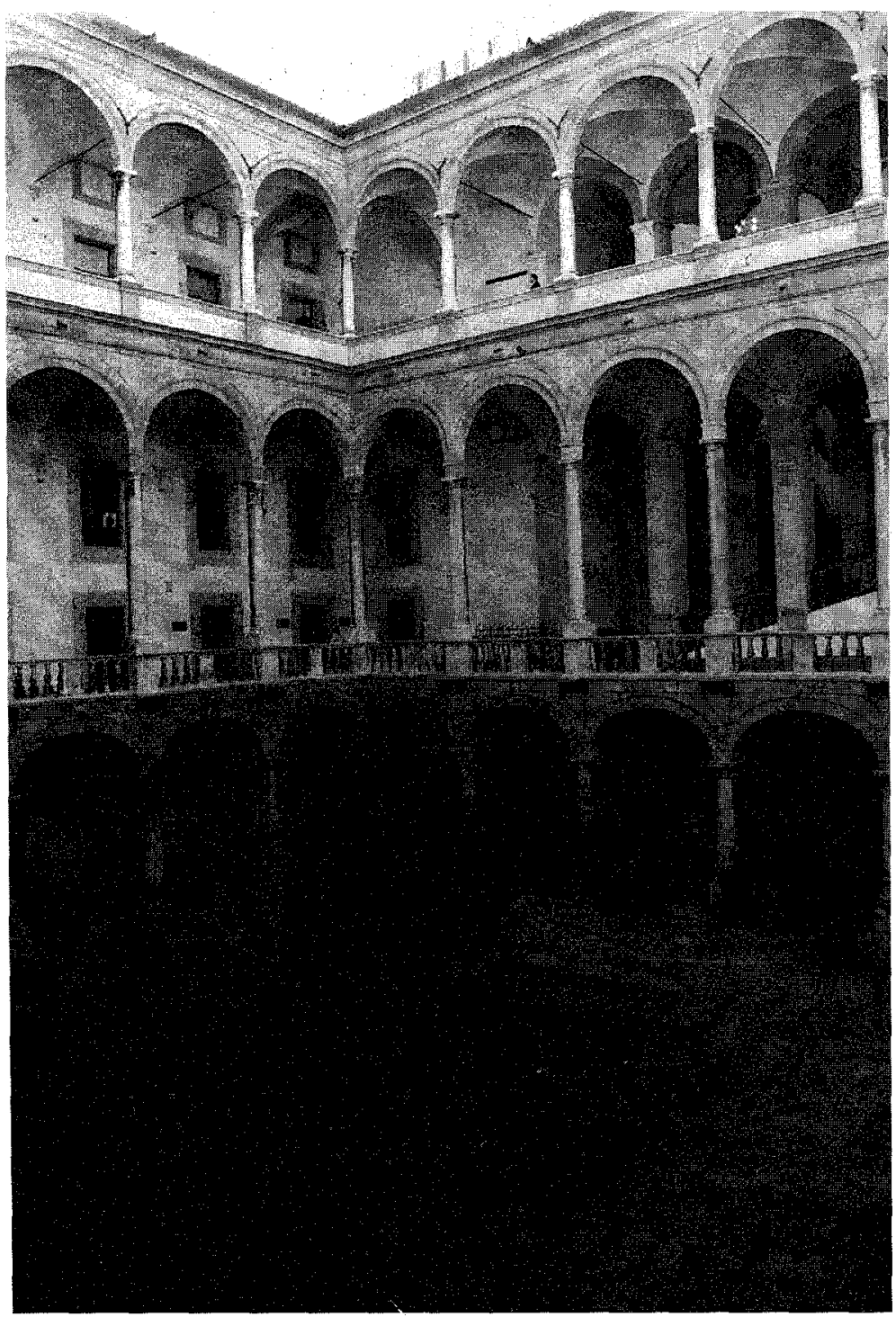

Fig. 5. Palermo. Palazzo Reale, cortile Maqueda. Dietro il loggiato, sulia parte destra dell'immagine, si intravede lo scalone. 


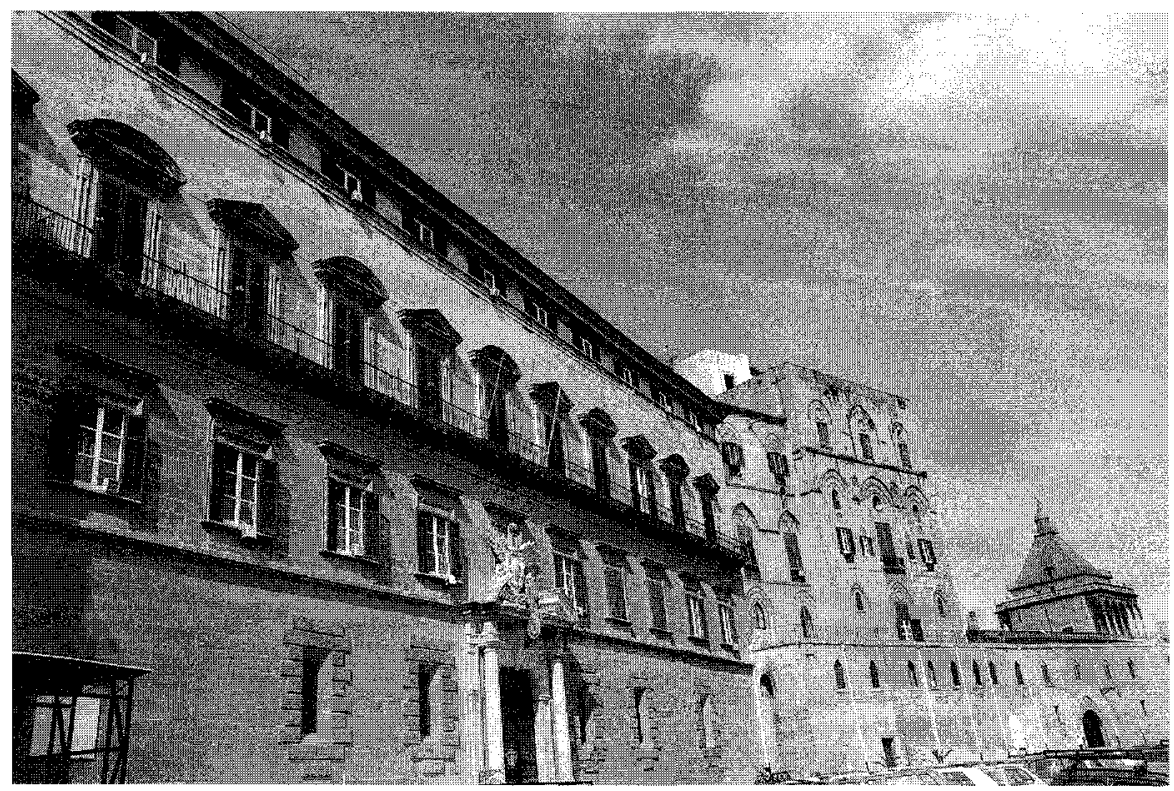

Fig. 6. Palermo. Palazzo Reale, fronte principale. All'estrema destra dellimmagine si intravede la terminazione di Porta Nuova.

Giorgi ${ }^{37}$. L'esigenza di far confluire su una stessa persona entrambe le cariche forse era stata avvertita, da parte della committenza viceregia e senatoriale, nel tentativo di creare per la Capitale una sorta di coordinamento tecnico o, comunque, di linea operativa uniforme riguardo alle numerose iniziative architettoniche e urbanistiche che nella seconda metà del Cinquecento avevano trasformato il volto della città. Tali convergenze, tuttavia, nel secolo successivo sembrano venire meno e in tal senso la vicenda dei Quattro Canti - le fastose quinte architettoniche costruite all'incrocio della via Toledo e della via Maqueda, destinate, fondamentalmente a contenere le statue dei re spagnoli - risulta emblematica, palesando l'avvenuta maturazione di una crisi ormai irreversibile all'interno delle forze edificatrici che proprio quel processo di trasformazione della città avevano innescato e gestito.

3o Per l'occasione venne pubblicato anche un libretto celebrativo dell'opera, redatto in occasione dell'inizio dei lavori, dedicato nella prima parte al Senato cittadino e nella seconda parte al viceré Villena, dove veniva descritto il progetto di Giulio Lasso. G.B. MARINGo, Fama dell'Ottangolo palermitano, Piazza Vigliena e Theatro del Sole, Palermo 1609. L'ingegnere Lasso è operativo in Sicilia soprattutto nel primo decennio del XVII secolo, dopo un soggiorno non molto fortunato in Spagna e in Bretagna durante gli ultimi anni del Cinquecento. A. CÁMARA MuÑOZ, Juan de Herrera 
Se, infatti, il primitivo progetto dell'opera redatto dall'ingegnere regio Giulio Lasso nel $1608{ }^{38}$ ne denuncia chiaramente il carattere di fondazione monarchica, i successivi interventi, operati tra il 1619 e il 1624 secondo precise direttive senatoriali, indicano inequivocabilmente la volontà di trasformare soprattutto l'apparato iconografico e scultoreo dell'opera ${ }^{39}$ in chia-

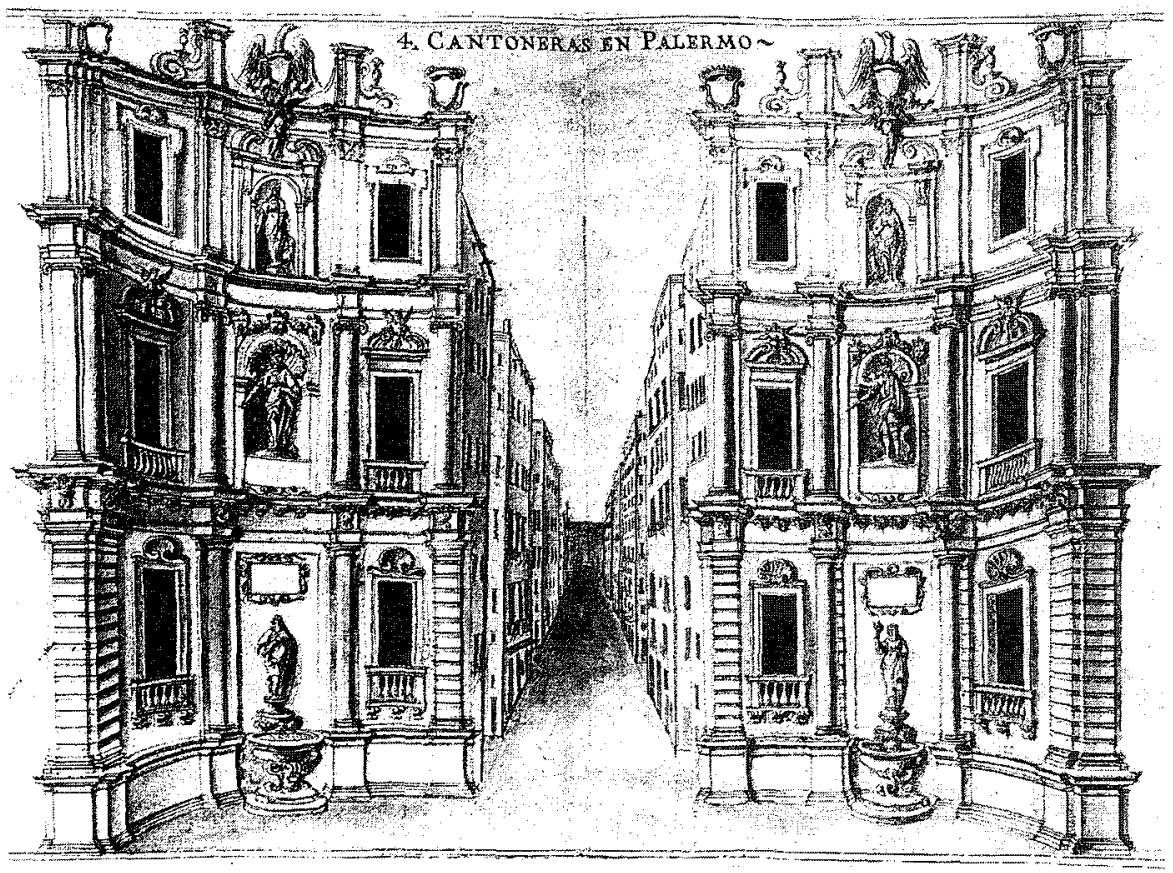

Fig. 7. Palermo. Veduta dei Quattro Canti dal Teatro geografico antiguo y moderno del Reyno de Sicilia (ms. de! 1686, Biblioteca Nazionale di Madrid-Ministero degli Affari Esteri di Madrid), pubblicato in V. CONSOLO, C. DE SETA, Sicilia Teatro del Mondo, Torino 1990, pág. 257, tav. 44.

y la arquitectura militar, in Juan de Herrera y la su Influecia, Atti del Convegno - Camargo 1992, Santander 1993, págs. 91-103, in particolare pág. 96, nota 46. Sulla figura di Giulio Lasso si vedano inoltre: A. Giuliana Alajmo, Architetti regi ..., cit., págs. 13-14; M. Fagiolo, M.L. Madonna, II Teatro ..., cit., appendice XIV, págs. 236-238; R. PAtricolo, F.S. Brancato, G. Fiducia, Giulio Lasso, Palermo 1991; L. Sarullo, Dizionario ..., vol. I, cit., págs. 252-254; R. Prescia, Storia e Restauri dell'Abbazia di San Martino delle Scale, Palermo 1995, págs. 44-46 e 112-113.

39 II nuovo progetto redatto nel 1619 prevedeva la sostituzione, allinterno delle nicchie del terzo ordine, dei simulacri reali con le statue delle sante protettrici della città e la realizzazione di quattro fontane da aggiungere al primo ordine. Su questa fase del cantiere abbiamo rinvenuto una vasta documentazione, pubblicata in M.S. DI FEDE, /l cantiere dei Quattro Canti a Palermo: il progetto del 1619, in «Annali del Barocco in Sicilia», 2, 1995, págs. 49-59. 
ve esclusivamente municipalistica. Non è un caso che il compito di apportare tali variazioni al progetto originario non sia stato affidato allo Smiriglio - ingegnere del Senato in carica però, allo stesso tempo, anche ingegnere regio - ma a Giovanni D'Avanzato.

La vicenda si concluse con un inevitabile compromesso fra le diverse soluzioni, ${ }^{40}$ mettendo fine alla contesa dei contenuti simbolici e rappresentativi sottesi al programma dell'opera da parte delle autorità senatoriali e viceregie, ma denunciando anche una frattura oramai incolmabile fra la società palermitana e l'autorità monarchica che sarebbe esplosa tragicamente nella rivolta del 1647.

${ }_{40}$ Nel 1630, durante il viceregno del duca di Alburquerque, si decise di intervenire nuovamente sulle quattro facciate, sostituendo i finestroni centrali del secondo ordine con quattro nicchie marmoree, per contenere le statue bronzee dei re spagnoli. F. MELI, Degli architetti ..., cit., págs. $305-470$, docc. 26,28 e 29 . In realtà saranno realizzate solamente due delle quattro statue bronzee per essere, inoltre, collocate in altri siti. I simulacri marmorei dei re saranno realizzati e collocati nelle nicchie soltanto fra il 1661 e 1663. M. FAGIOLO, M.L. MAdONNA, II Teatro ..., cit., pág. 71. 
